\title{
Wirtschaftswachstum: Freund oder Feind zukünftiger Generationen?
}

\author{
Ein zentraler ethischer Aspekt der Nachhaltig- \\ keitsdebatte ist die Frage nach der Gerechtigkeit \\ zwischen den Generationen. Wir alle wollen, \\ das es auch unseren Kindern gut geht. Aber \\ kann Wirtschaftswachstum dazu beitragen? \\ Von Dominic Roser
}

G enerationengerechtigkeit befasst sich mit Pflichten, die wir gegenüber zukünftigen Generationen haben. Ist die Förderung von Wirtschaftswachstum eine solche Pflicht? Oder ist es im Gegenteil der Verzicht darauf? Wenn man der Debatte um diese Frage in den verschiedenen Kontexten Gehör schenkt, so stößt man auf eigenartige Spannungen.

\section{Wachstum und Gerechtigkeit}

Der Gerechtigkeitstheoretiker John Rawls behandelt Generationengerechtigkeit anhand der Frage um "gerechtes Sparen”: Wie viel dürfen die heute lebenden Menschen, die Gegenwärtigen, vom vorhandenen Kapital konsumieren und wie viel sollen sie zu Gunsten späterer Generationen, der Zukünftigen, sparen. Sparen heißt immer auch investieren und somit potenzielles Wachstum. Die Diskussion um das gerechte Sparen trägt also in gewissem Sinne der Befürchtung Rechnung, dass die Gegenwärtigen zu wenig für das Wirtschaftswachstum tun könnten.

Ganz anders ist die typische Befürchtung, wenn die Diskussion um Generationengerechtigkeit im Kontext ökologischer Fragen geführt wird. Hier besteht die Befürchtung, dass Wirtschaftswachstum im Interesse der Gegenwärtigen liegt und wegen der zerstörten Lebensgrundlagen den Interessen der $\mathrm{Zu}$ künftigen zuwiderläuft. Gerechtigkeitsforderungen sind damit beschäftigt, dass sich die Gegenwärtigen nicht für zu viel Wirtschaftswachstum entscheiden.

Nochmals anders sieht die Diskussion im Bereich der Altersvorsorge aus. Wirtschaftswachstum wird in diesem Kontext als eine Win-win-Strategie betrachtet. Es bringe Vorteile für Zukünftige, weil der gewachsene Wohlstand die Verteilkämpfe verringere, aber auch für Gegenwärtige, zum Beispiel in Form wachsender Beschäftigung. Diese verschiedenen Stoßrichtungen animieren dazu, eine allgemeine Antwort auf folgende
Grundfrage zu suchen: Fordert Generationengerechtigkeit von uns weniger oder mehr Wirtschaftswachstum? Um weiterzukommen, muss diese Grundfrage in drei Teilfragen aufgetrennt werden. Dazu gehört eine normative Frage. Müssen die Gegenwärtigen dafür sorgen, dass es den Zukünftigen besser geht? Ebenso wichtig ist eine empirische Frage. Trägt Wirtschaftswachstum dazu bei, dass es den Menschen besser geht? Drittens spielt eine weitere normative Frage eine Rolle. Angenommen, die zweite Frage liefert keine eindeutige Antwort, zum Beispiel weil Wirtschaftswachstum die Lebensqualität der Gegenwärtigen erhöht, die Umweltqualität für die Zukünftigen jedoch vermindert. Dürfen diese verschiedenen Auswirkungen des Wirtschaftswachstums in einer Gesamtbeurteilung gegeneinander aufgewogen werden? Im Folgenden sollen zu den drei Fragen kurze Bemerkungen folgen.

Zuerst aber noch eine Vorbemerkung. Wirtschaftswachstum heißt, dass das reale Bruttoinlandsprodukt (BIP) steigt, wobei das BIP ein relativ enges Maß für den Wert der produzierten Güter und Dienstleistungen ist. Als Voraussetzung für diesen Text wird angenommen, dass das BIP nicht schon an sich einen Wert darstellt, sondern höchstens instrumentell wertvoll ist. Der Einfachheit halber wird hier angenommen, dass Lebensqualität das ist, was wirklich zählt. Natürlich gibt es daneben viele andere Kandidaten für intrinsisch Wertvolles, wie zum Beispiel Glück aller empfindenden Kreatur, Natur oder Schönheit.

\section{Eine bessere Zukunft}

Zur ersten Frage kann Folgendes gesagt werden: Wirtschaftswachstum wird gefordert, damit es der Menschheit je länger je besser gehe. Wird vorausgesetzt, dass Wirtschaftswachstum tatsächlich die Lebensqualität erhöht, dann bleibt immer noch die folgende Frage: Haben die Gegenwärtigen überhaupt die Pflicht dafür zu sorgen, dass es den Zukünftigen besser geht? Unsere Alltagsintuition sieht eine positive Sparrate, also ein Verzicht der Gegenwärtigen zugunsten eines höheren Lebensstandards der Zukünftigen, typischerweise als angebracht an. Diese scheinbar selbstverständliche Pflicht einer dauernden Verbesserung der menschlichen Situation ist aber gar nicht so einfach zu begründen.

Schauen wir uns kurz an, was drei verschiedene Perspektiven - Utilitarismus, Egalitarismus und die Idee der Nachhaltigkeit - dazu beitragen. Der Utilitarist hat als Einziger keine großen Schwierigkeiten, eine positive Sparrate zu begründen. Die Idee des Utilitarismus besteht darin, den über die verschiedenen Generationen summierten Nutzen zu maximieren. Und $\rightarrow$ 


\section{„Für entwickelte Länder ist es zweifelhaft, ob Wirtschaftswachstum instrumentellen Wert für die Steigerung der Lebensqualität hat.“}

da eine Konsumeinheit, auf die heute verzichtet wird und die stattdessen investiert wird, morgen mehr als eine Einheit Konsum ermöglicht, liegt es auf der Hand, dass eine Maximierung über die Zeit Sparen erfordert. Wie viel genau zur Nutzenmaximierung gespart werden soll, beschäftigt ökonomische Optimal-growth-Modelle.

Für den Egalitaristen hingegen ist es schwierig zu begründen, weshalb sich Gegenwärtige zugunsten Zukünftiger einschränken sollten. Die Forderung stellt offensichtlich eine antiegalitaristische Forderung dar. John Rawls mildert diese kontraintuitive Folgerung des Gleichheitsanliegens ab, indem er von früheren Generationen, denen es im Vergleich zu späteren schlechter geht, tatsächlich Sparen fordert. Sparen ist laut Rawls aber nur so lange erforderlich, bis die ökonomischen Grundlagen dafür geschaffen sind, dass in einer Gesellschaft gerechte Strukturen stabil bestehen können.

Auch Nachhaltigkeitsforderungen verlangen typischerweise nicht, dass es den Zukünftigen besser gehen muss, sondern nur, dass die Gegenwärtigen so leben, dass es den Zukünftigen nicht schlechter geht oder dass die Zukünftigen ihre Bedürfnisse befriedigen können. Zusammenfassend lässt sich sagen, dass die Forderung, es den Zukünftigen nicht schlechter gehen zu lassen, viel stärker präsent ist, als die Forderung, eine bessere Welt zu hinterlassen. Als Nachbemerkung bleibt zu sagen, dass die Frage „Soll es den zukünftigen Generationen besser gehen?” natürlich viel Spielraum offen lässt, denn sie beantwortet noch nicht, wem es in den zukünftigen Generationen besser gehen soll (Wolf 2006).

\section{Der Wert des Wirtschaftswachstums}

Die zweite Frage betrifft den Wert des Wirtschaftswachstums. Unabhängig von der ersten Frage können wir uns fragen, ob denn Wirtschaftswachstum tatsächlich zu einer besseren Welt beitragen würde und mehr Lebensqualität zur Folge hätte. Diese Frage ist schon deshalb relevant, weil viele ja eine bessere Welt hinterlassen wollen.

In einem ersten Beantwortungsschritt kann ohne Einbezug der Umweltproblematik gefragt werden, ob mehr BIP mehr Lebensqualität bedeutet. Diese Frage findet leider immer noch zu wenig wissenschaftliche Beachtung. Eine begrüßenswerte Ausnahme sind die happiness economics, die sich mit einem zentralen Bestandteil der Lebensqualität, dem Glück, beschäftigen. Empirische Resultate haben wiederholt bestätigt, dass zumin- dest in entwickelten Ländern Wirtschaftswachstum die Menschen nicht glücklicher macht. Es ist anzunehmen, dass dies für Menschen unter einer absoluten Armutsgrenze anders aussieht, was allerdings noch nicht durch Studien belegt wurde (Easterlin 2002).

Wenn man in einem zweiten Schritt die Umweltproblematik einbezieht, gerät Wirtschaftswachstum als geeignetes Mittel zur Steigerung der Lebensqualität unter zusätzlichen Beschuss. Dies ist erstens der Fall, weil Wirtschaftswachstum durch die Umweltbelastung seine eigenen Grundlagen zu untergraben droht. Und zweitens ist nicht zu vergessen, dass viele Ethiker den Wert der Natur nicht nur in ihrer Rolle als instrumentellen Wert bei der Generierung von Lebensqualität sehen. Vielmehr sehen sie die Natur auch als intrinsischen Wert, dessen Verminderung einen weiteren Minuspunkt für das Wirtschaftswachstum darstellt.

Allerdings muss nach diesen wachstumskritischen Bemerkungen sogleich ein Einschub folgen. Jede angewandte Ethik sollte neben idealen Normen auch Praxisnormen bereitstellen. Praxisnormen sind für reale Menschen handlungsleitend, also für Menschen, deren Willensstärke und deren kognitive Fähigkeiten beschränkt sind (Birnbacher 1988: 16). Die Unterscheidung zwischen idealen Normen und Praxisnormen ist für die Diskussion um Wirtschaftswachstum wichtig, weil in der realen Welt offensichtlich jegliche Bereitschaft fehlt, auf Wachstum zu verzichten, sowohl in Entwicklungs- als auch in Industrieländern. Beispielhaft ist Bushs Verweis auf das Wirtschaftwachstum bei der Ablehnung des Kyoto-Protokolls. Für eine praxisrelevante Ethik besteht zurzeit sehr wenig Spielraum im Spektrum "wenig versus viel Wachstum“. Immerhin besteht ein gewisser Spielraum zwischen sauberem und dreckigem Wachstum.

Wichtig sind solche pragmatischen Aspekte auch, wenn die Nützlichkeit des Wirtschaftswachstums mit der Erweiterung der Verteilungsspielräume begründet wird. Aus der Perspektive idealer Normen ist eine solche Begründung sehr schwach. Wenn zum Beispiel wegen des demographischen Wandels der Kuchen zwischen Rentenbeziehern und Rentenzahlern neu aufgeteilt werden muss, so ist bei der Suche nach idealen Normen alleine die Frage wichtig, wie groß der Kuchen ist und wie er verteilt wird. Gänzlich irrelevant hingegen ist die Feststellung, dass bei Umverteilungen mit gleichzeitig wachsendem Kuchen die Verlierer bloß in Kauf nehmen müssen, dass ihr Stück im Gegensatz zu den Gewinnern nicht wächst. Wohingegen bei Umverteilungen mit gleich bleibender Kuchengröße das Stück der Verlierer schrumpft. Aus der Perspektive von Praxisnormen hingegen ist es sehr relevant, dass eine Erweiterung der Verteilungsspielräume großen Einfluss auf die Durchsetzbarkeit von Umverteilungen mit sich bringt.

Zusammenfassend lässt sich sagen, dass es für entwickelte Länder zweifelhaft ist, ob Wirtschaftswachstum instrumentellen Wert für die Steigerung der Lebensqualität hat. Dies gilt sowohl unter Einbezug des Zusammenhangs zwischen Wirtschaftswachstum und Umweltqualität als auch ohne dessen 
Einbezug. Allerdings hat eine solche Aussage beschränkten praktischen Wert, da der Drang zum Wachstum in der politischen Arena eine unheimliche Wucht hat.

\section{Aufwiegen verschiedener Konsequenzen}

Die dritte Frage lässt sich wie folgt beantworten. Wirtschaftswachstum kann verschiedene Konsequenzen haben. Es kann zum Beispiel materiellen Wohlstand bringen, Umweltqualität verringern oder mit Staatsschulden einhergehen. Inwiefern dürfen diese verschiedenen Effekte miteinander aufgerechnet werden? Darf man zum Beispiel Defizite in der Generationenbilanz wie übermäßige Staatsschulden mit einer verbesserten Ökobilanz aufwiegen? Auch wenn die grundsätzliche Antwort wohl ja lautet, so gibt es doch zwei Probleme zu beachten.

Das erste Problem erlangte Prominenz im Rahmen der Debatte um schwache und starke Nachhaltigkeit. Starke Nachhaltigkeit unterscheidet zwischen menschlich produziertem Kapital und Naturkapital und verlangt, dass von beidem genügend zu bewahren sei. Schwache Nachhaltigkeit hingegen fordert bloß, dass den Zukünftigen insgesamt genügend Kapital zur Verfügung zu stehen habe, welcher Art das Kapital auch immer sei. Ein Vertreter im Sinne der starken Nachhaltigkeit, der sich gegen die Verrechenbarkeit der verschiedenen Auswirkungen des Wirtschaftswachstums wendet, kann zwei Strategien fahren. Er kann einerseits eingestehen, dass Lebensqualität das einzig intrinsisch wertvolle ist. Um dann aber als empirische Aussage zu behaupten, dass zukünftige Generationen zu diesem Zweck von verschiedenen Gütern, wie zum Beispiel menschlich produziertem Kapital und Naturkapital, genug zur Verfügung stehen müsse, da die beiden Arten von Kapital schlecht substituierbar seien. Er kann andererseits aber auch ohne diese empirische Behauptung auskommen, und dafür als normative Aussage behaupten, dass neben Lebensqualität auch die Natur intrinsisch wertvoll sei und der moralisch akzeptable Spielraum für Güterabwägung zwischen den zwei Werten Lebensqualität und Natur beschränkt sei. Hinzu kommt ein zweites Problem, das gegen allzu simples Aufwiegen der verschiedenen Auswirkungen von Wirtschaftswachstum spricht, nämlich dass die Nachwelt aus verschiedenen Menschen zu verschiedenen Zeiten besteht. Zum Beispiel mag ein schnelles und klimaschädigendes Wachstum in China einer Chinesin in 20 Jahren zum Vorteil gereichen, einem Afrikaner in 80 Jahren aber zum Nachteil. Auch wenn die Gesamtmenge an Nutzen, die wir der Nachwelt hinterlassen, die Kosten überwiegen sollte, so ist damit alleine unser Verhalten noch nicht legitimiert. Und zwar dann nicht, wenn Nutzen und Kosten je verschiedene Menschen betreffen.

Besonders kritisch ist das Aufrechnen von positiven und negativen Konsequenzen des Wachstums, wenn diese sowohl gleichzeitig Lebende als auch nach uns Lebende betreffen. Letzterer Fall, das heißt der Fall nicht-überlappender Generationen, bringt einige Schwierigkeiten für eine Theorie intergenerationeller Gerechtigkeit mit sich. Erstens hängt es bei nach uns Le- benden stark von uns ab, welche Individuen überhaupt existieren werden. Es ist aber problematisch, einem Individuum das Recht auf eine bestimmte Behandlung unsererseits zu zusprechen, wenn seine Existenz überhaupt erst von dieser Behandlung abhängt. Zweitens existieren zukünftige Menschen noch nicht, was die Frage aufwirft, ob nicht-existente Menschen überhaupt Rechte haben können. Drittens ist die Beziehung zu den nach uns Geborenen einseitig. Sie können uns weder beeinflussen noch mit uns kooperieren - gerade solche gegenseitigen Beziehungen stellen aber einen zentralen Bestandteil vieler Gerechtigkeitstheorien dar. Diese Komplikationen haben zur Folge, dass Gerechtigkeit zwischen nicht-überlappenden Generationen andere, typischerweise geringere Forderungen an Gegenwärtige stellt als Gerechtigkeit zwischen überlappenden Generationen.

Zusammenfassend lässt sich sagen, dass es nicht völlig belanglos ist, in welcher Form wir der Nachwelt Werte hinterlassen - ob zum Beispiel in Form geringerer Staatsschulden, besserer Umweltqualität oder eines höheren Bildungsniveaus. Erstens zeigt uns die Diskussion um schwache und starke Nachhaltigkeit, dass berechtigte Güterabwägungen ihre Grenzen haben. Und zweitens sagen uns intergenerationelle Gerechtigkeitstheorien, dass es darauf ankommt, wem wir innerhalb der Nachwelt Werte hinterlassen - insbesondere, ob wir sie gleichzeitig oder nach uns lebenden Generationen hinterlassen.

\section{Eine offene Frage}

Zusammenfassend für den ganzen Text lässt sich sagen, dass zwei Punkte zumindest zweifelhaft sind: Erstens, ob Wirtschaftswachstum zu einer besseren Welt beiträgt und zweitens, ob Generationengerechtigkeit überhaupt dazu verpflichtet, eine bessere Welt zu hinterlassen. Folglich ist es zumindest zweifelhaft, dass Generationengerechtigkeit Wirtschaftswachstum fordert. Als Nachbemerkung sei hinzugefügt, dass uns dies natürlich nicht daran hindern muss, aus einem Motiv des Wohlwollens heraus eine bessere Welt für unsere Nachfahren anzustreben. Inwiefern Wirtschaftswachstum dazu ein geeignetes Mittel ist, bleibt eine offene Frage. Und eine Wichtige dazu.

\section{Literatur}

Birnbacher, D.: Verantwortung für zukünftige Generationen. Stuttgart 1988. Easterlin, R. (ED): Happiness in Economics. Cheltenham 2002.

Gosseries, A.: The Egalitarian Case Against Brundtland's Sustainability. In: GAIA 14/1 2005.

Parfit, D.: Reasons and Persons. Oxford 1984.

Wolf, C.: Anthropogenic Climate Change and Intergenerational Justice. In: Meyer, L./ Gosseries, A. (Hrsg.): Theories of Intergenerational Justice (in Vorbereitung). Oxford 2006.

\section{AUTOR + KONTAKT}

Dominic Roser ist Mitarbeiter am Institut für Philosophie der Universität Bern.

Universität Bern, Länggasseetr. 49a, CH-3012 Bern. Tel. 0041/(0)31/371-1651,

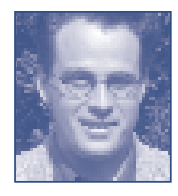

E-Mail: dominic.roser@vwi.unibe.ch 
(c) 20I0 Authors; licensee IÖW and oekom verlag. This is an article distributed under the terms of the Creative Commons Attribution Non-Commercial No Derivates License (http://creativecommons.org/licenses/by-nc-nd/3.o/), which permits unrestricted use, distribution, and reproduction in any medium, provided the original work is properly cited. 